\title{
IMPLEMENTATION OF IOT ON VEHICLE PAYLOAD MONITORING
}

\author{
Jeeva C, Ganesh K S, Gowtham A, Barathwaaj D. \\ Department of Mechanical Engineering. \\ Sri Eshwar College of Engineering, Coimbatore, India
}

\begin{abstract}
The continuous development and evolution of electric vehicle causes the electric car manufacturers to face new challenges. Now, the lithium-ion batteries are widely used in electric vehicles which has high current discharge rate. During overloading, electric motor draws large amount of current which leads to degradation in battery life and overheating. Most of the time, the user is unaware of vehicle loading. This paper discusses the method to overcome this issue by estimating vehicle load on real time and alerting the use of vehicle. Using load cell to measure the data and send the data to the cloud by means of Amazon Web Services (AWS). When the vehicle is overloaded, a notification sent to the user. Realtime loading data is shown on dashboard. Using evolving Technologies like IoT makes easy to storing the data on cloud. This enables service engineers to evaluate and improve vehicle, based on working conditions for future needs. This can also be used for future needs like reliable range prediction of electric vehicle and autonomous vehicle driving system.
\end{abstract}

Keywords - Electric vehicles, hybrid electric vehicles, batteries, internet of things.

\section{INTRODUCTION}

Many countries have turned their interest towards the electric vehicles over the conventional fuel driven cars for its ecofriendly and absence of various systems like lubrication, cooling and exhaust system. But this put forth new problems that were needed to be addressed. One of such problem is battery failure and battery life reduction. These issues were mainly occurred when the user overload their electric vehicle. Most of the situations, the user will not be aware of the vehicle payload and may not always be using weight bridge to check weight regularly. This problem can be overcome by measuring pay load on Realtime and notifying the user if its overloaded. This paper will explain a simple methodology of the implementation of load measurement in real time and storing the loading data on cloud. Model uses load cells to measure the payload and the loading data is sent to raspberry $\mathrm{Pi}$. This data is then logged to the cloud. This system will notify the user when the load exceeds the pre-set maximum load. Thus, preventing the user from exploiting the battery and maintaining its life cycle.

\section{METHODOLOGY}

This work explains about the method of measuring the payload in an electric vehicle and store the data in Amazon IoT cloud. Strain gauge (load cell) is used to measure the load and corresponding signal is sent to amplifier and Analog-to-digital converter. The converter sends the digital data corresponding to the load to the raspberry pi. Based on the predetermined loading condition, raspberry pi checks the data and send the loading data if its permissible limit and send a notification to the user if the data is high. This method prevent user from overloading his electric vehicle which will drain the battery faster and reduce its life cycle. Battery warranty is void if the user overloads the battery. The service engineer may determine battery warranty state by checking the logged data in cloud.

\section{COMPONENTS AND FUNCTIONS:}

Various components used in the model are,

- Raspberry Pi,

- Strain gauge (load cell),

- Analog to digital converter and amplifier.

A. Raspberry Pi-

Raspberry Pi is a small single board computer, which weighs only $50 \mathrm{~g}$. The Raspberry Pi 3 B+ model has 512 MB RAM, runs on ARMII CORTEX A53 processor. The frequency of operation of the processor is $700 \mathrm{MHz}$. It has various interfacing ports like USB 2.0, HDMI, GPIO (digital), display connector and camera connector. It can be powered by a simple $5 \mathrm{~V}$ battery minimum of $700 \mathrm{mah}$. It has built in $\mathrm{W}-\mathrm{Fi}$ and Bluetooth for networking.

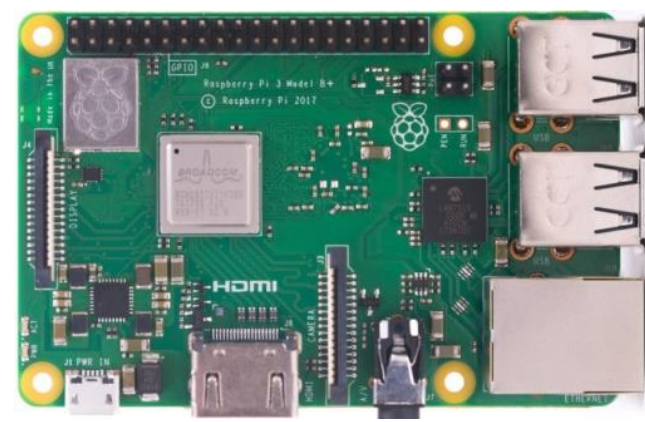

Fig. 1. Raspberry pi 


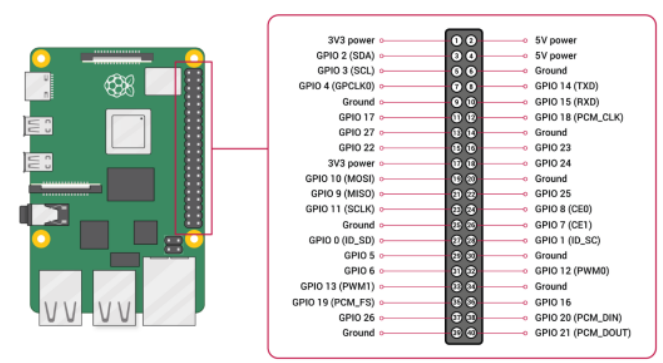

Fig. 2. Pinout

\section{B. Strain gauge-}

It measures mechanical stress caused by deformation due to application of load and providing corresponding analog signals. There are two types of resistive strain gauge in which metallic strain gauge are selected. The resistance of the strain gauge changes with respect to the deformation formed due to the applied load.

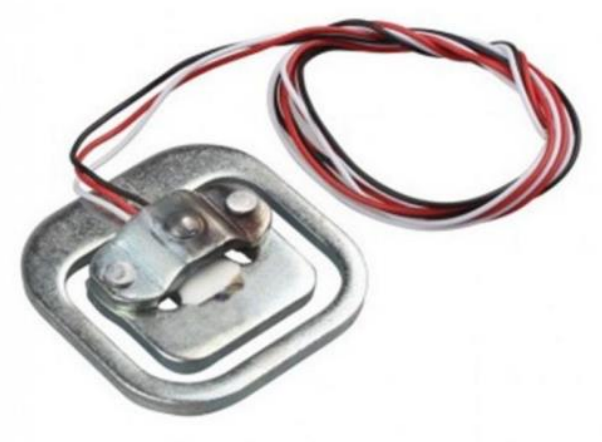

Fig. 3. Strain Gauge

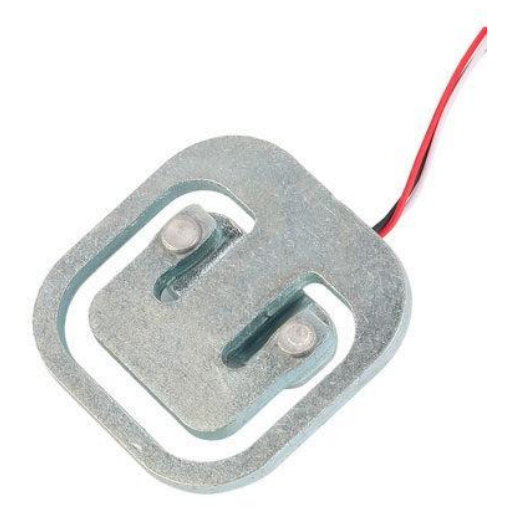

Fig. 4. Strain Gauge

\section{Analog to digital converter and amplifier-}

Signals produced by resistive strain gauge is change in current. This signal is very small and hence the signal is first amplified and the converted into digital values using HX711 circuit board.

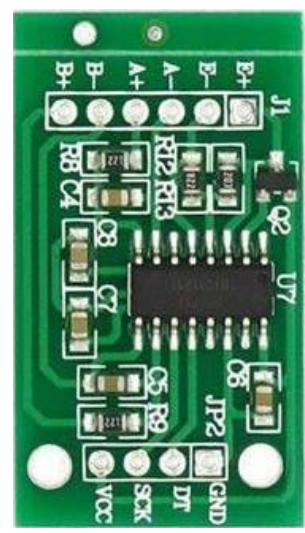

Fig. 5. ADC

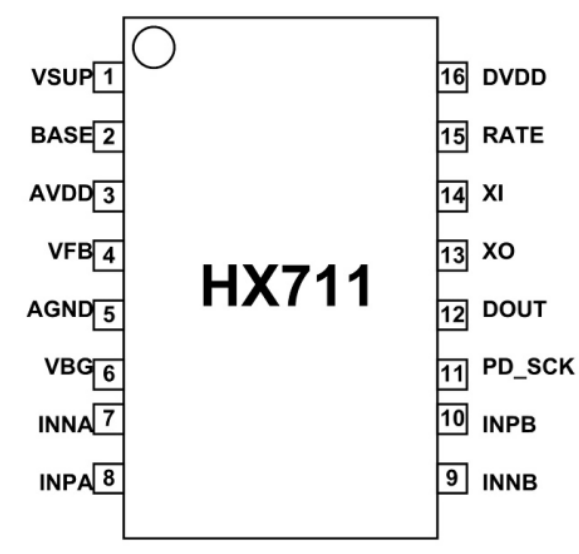

Fig. 6. HX711

\section{Connections And Working Of The System}

- Load cell is connected to the HX711 analog to digital converter and amplifier.

- $5 \mathrm{~V}$ power is given to HX711 and the data pins are connected to digital pins of the Raspberry pi.

- Load cell measures the payload and produces corresponding signal to HX711.

- HX711 amplify the signal and convert the amplified signal to digital data.

- This data is sent read by raspberry pi and logged.

- The data is also sent to Amazon Web Services cloud.

- If the payload value exceeds the pre-set range, notification is sent to the user. 


\section{International Journal of Engineering Applied Sciences and Technology, 2021 \\ Vol. 5, Issue 11, ISSN No. 2455-2143, Pages 222-225 \\ Published Online March 2021 in IJEAST (http://www.ijeast.com)}

- Pay loading data is displayed on dashboard on real time and also can be viewed in AWS cloud remotely.

\section{FLOW CHART}

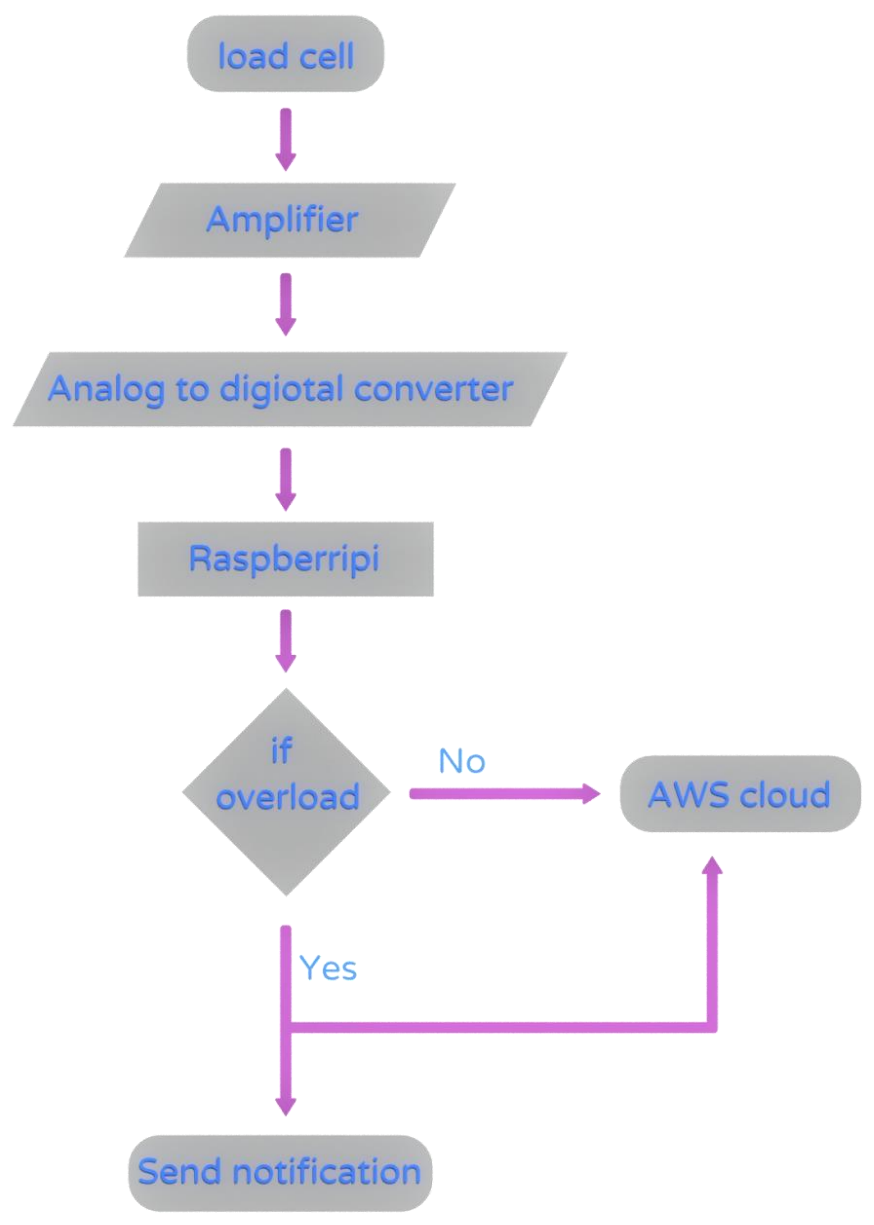

Fig. 7. Flow chart

\section{REVIEW}

Several cities of North America are within the changeover period to rapidly replace conventional vehicles with hybrid plug-in vehicles then to EVs [3]. EVs has more benefits in terms of cost and convenience. Unlike internal combustion engine (ICE) vehicles, the maintenance costs do not exist in an EV [4]. To evaluate the lateral and longitudinal motion in the context of the vehicle dynamics stability. A very precise detection of the vehicle mass makes a more reliable control performance of AVs [9] and EVs possible [5]. The patent application [6] of the Volvo Truck Corporation is one example for a system that will use the dynamical method. At first, this system approach will monitor and compare the acceleration behaviour of different period's respectively different operating conditions. After this, the system will use a Kalman filter and calculate as well as compare the different driving resistances. Based on this comparison, the system will be able to estimate the vehicle mass. Volvo states that this system has the potential to optimize brake systems or to realize efficient driving strategies depending on the current vehicle mass.

\section{CONCLUSION}

This paper clearly shows the necessity for real time payload monitoring in an electric vehicle. Integrating IoT will enable new possibilities like overload notification and loading data log. Thus, user may be well aware of the load on his vehicle and can prevent his vehicle from overloading. This also enables service engineers to check for clean ability of warranty for the user's working conditions

\section{REFERENCE}

[1] Heiko Fechter and Benedikt Schmulling, "Survey of Current Vehicle Mass Estimators in the Context of Future Mobility", pp.81-84, 2017.

[2] Heiko Fechtner, Thomas Teschner, and Benedikt Schmuelling, Member IEEE, "Range Prediction for Electric Vehicles: Real-Time Payload Detection by Tire pressure Monitoring”, pp.767-772 , 2015.

[3] P. Sivaraman and C. Sharmeela, "IoT-Based Battery Management System for Hybrid Electric Vehicle", pp.0204, 2020.

[4] Md Masud Rana: - "Iot Based Electric Vehicle State Estimation and Control Algorithms", pp.02, 2019.

[5] E S Xydas, C E Marmaras, L M Cipcigan, A S Hassan, N Jenkins, "Electric Vehicle Load Forecasting using Data Mining Method", pp.02-04, 2013.

[6] Mahammad A. Hannan, Md. Murshadul Hoque, Yushaizad Yusof, And Pin Jern Ker, "State-of-the-Art and energy Management System of Lithium-Ion Batteries in Electric Vehicle applications”, pp.19364-019370, 2018.

[7] Cleber M. de Morais, "An IoT Sensor and scenario survey for Data Researchers”, pp. 01-02, 2019.

[8] Xuebing Han, Minggao Ouyang, Languang Lu, Jianqiu Li, "A Comparative study of commercial Lithium ion Battery Cycle life in Electric Vehicle: Capacity Loss Estimation”, pp.660-670, 2013.

[9] Alessio Botta, Walter de Donato, Valerio Persico, Antonio Pescape, "Integration of Cloud Computing and Internet of Things", pp.06-07, 2015.

[10] George F. Savari, Vijayakumar Krishnasamy, Jagabar Sathik, Ziad M. Ali, Shady H.E. Abdel Aleem, "Internet of Things based real-time vehicle load forecasting and charging station recommendation”, pp.12-13, 2019.

[11] Partha Pratim Ray, "A Survey of IoT Cloud Platforms", pp.06, 2017.

[12] K. S. Mohamed, "IoT Cloud Computing, Storage, and Data Analytics”, pp.72, 2019. 
[13] Luciano Barreto , Antonio Celesti , Massimo Villari , Maria Fazio, Antonio Puliafito, "An Authentication Model for IoT Clouds", pp.1033-1035, 2015.

[14] Lu Zhoua, Xiong Lib , Kuo-Hui Yeha , Chunhua Sua , Wayne Chiuc, "Lightweight IoT-based authentication scheme in cloud computing circumstance", pp.03-07, 2018.

[15] Hong-Linh Truong and Schahram Dustdar, "Principles for Engineering IoT Cloud Systems”, Pp.02-03, 2015.

[16] Pankaj Ganguly, "Selecting the right IoT Cloud Platform", Pp.318-320, 2016.

[17] Rajkumar Buyya, Chee Shin Yeoa, Srikumar Venugopal, James Broberg, Ivona Brandic, "Future Generation Computer Systems", pp.13-14, 2008.

[18] Masaki Yoshio, Ralph, Brodd, Akiya Kozawa, "LithiumIon Batteries", pp.xviii, 2009.

[19] Khizir Mahmud, Hossain, and Jayashri Ravishankar, "Peak-Load Management in Commercial Systems With Electric Vehicles", pp.06-07, 2017.

[20] Jaewan Kim, Jinwoo Oh, Hoseong Lee, "Review on battery thermal management system for electric vehicles", pp.01-02, 2018.

[21] Bogdan Cristian Florea, Dragos Daniel Taralunga, " IoT for Smart Electric Vehicles Battery Management”, pp.01$02,2020$. 